\title{
ARMAZENAMENTO REFRIGERADO DE UVAS CVS. TARDIA DE CAXIAS E DONA ZILÁ
}

\author{
COLD STORAGE OF 'TARDIA DE CAXIAS' \\ AND 'DONA ZILÁ’ TABLE GRAFES
}

\author{
Auri Brackmann ${ }^{1}$ Sérgio Miguel Mazaro ${ }^{2}$ \\ Alessandro Jaquiel Waclawovsky ${ }^{3}$
}

\section{RESUMO}

\begin{abstract}
O objetivo deste trabalho foi avaliar condições de armazenamento sobre as características físico-químicas e organolépticas e tratamentos pós-colheita para o controle de podridões em uvas de mesa cvs. Tardia de Caxias e Dona Zilá. As condições de armazenamento testadas foram: $-1,0^{\circ} \mathrm{C},-0,5^{\circ} \mathrm{C}$ ou $+0,5^{\circ} \mathrm{C}$ com $97 \%$ de umidade relativa (UR) $e-0,5^{\circ} \mathrm{C}$ com $93 \%$ de UR. Os tratamentos pós-colheita para controle de podridões foram: cloreto de cálcio $\left(\mathrm{CaCl}_{2}-0,5 \%\right)$ aplicado por imersão; dióxido de enxofre $\left(\mathrm{SO}_{2}\right)$ aplicado semanalmente através de $4 \mathrm{~g}$ de metabissulfito de sódio $\left(\mathrm{Na}_{2} \mathrm{~S}_{2} \mathrm{O}_{5}\right) ; \mathrm{CO}_{2}(5 \mathrm{kPa})$ durante todo o período de armazenamento e; tratamentos intermitentes com $\mathrm{CO}_{2}$ $(20 \mathrm{kPa})$, aplicados semanalmente durante 24 horas. $O$ delineamento experimental foi o inteiramente casualizado com três repetições. As avaliações foram realizadas aos 35 dias de armazenamento e após dois dias de exposição desses frutos à temperatura ambiente $\left( \pm 20^{\circ} \mathrm{C}\right)$. As cultivares Tardia de Caxias e Dona Zilá não apresentaram sensibilidade à baixa temperatura de armazenamento. A umidade relativa de $97 \%$ diminuiu a perda de peso, atém de reduzir o escurecimento do ráquis e o degrane das bagas. $\mathrm{O}$ tratamento pós-colheita com $\mathrm{CaCl}_{2}$ causou danos à epiderme, aumentando as podridões. $\mathrm{O} \mathrm{SO}_{2}$ não influenciou a incidência de podridões. Já a utilização de altas concentrações de $\mathrm{CO}_{2}$ diminuiu ligeiramente as perdas por podridões. As cvs. Tardia de Caxias e Dona Zilá possuem boa capacidade de armazenamento, podendo ser conservadas sob refrigeração na temperatura de $-1,0^{\circ} \mathrm{Ca}+0,5^{\circ} \mathrm{C}$.
\end{abstract}

Palavras-chave: Vitis vinifera, pós-colheita, frigoconservação.

\section{SUMMARY}

The objective of the present work was to evaluate storage conditions and postharvest treatments on the quality of Tardia de Caxias' and 'Dona Zilá' table grapes. Temperatures of
$-I, 0^{\circ} \mathrm{C},-0,5^{\circ} \mathrm{C}$ or $+0,5^{\circ} \mathrm{C}$ were combined with relative humidities of 93 or $97 \%$. Postharvest treatments were calcium chloride $\left(\mathrm{CaCl}_{2}-0,5 \%\right)$, sulfur dioxide $\left(\mathrm{SO}_{2}\right)$ or high $\mathrm{CO}_{2}$ concentrations $(5 \mathrm{kPa})$, during the whole storage period or $2 \mathrm{OkPa} \mathrm{CO}_{2}$ applied intermittentely every week for 24 hours. Table grapes were evaluated after 35 days of storage and after two more days of shelf life $\left(i 20^{\circ} \mathrm{C}\right)$. Both cultivars are not sensitivo to $h w$ temperature during the storage. The $97 \% \mathrm{RH}$ treatment decreased the weight loss, browning of the ráquis and abscission of the fruitlets. Calcium chloride treatment caused damage to the skin, increased rot incidence. Sulfur dioxide treatment was not effective in the rot contrai. High concentrations of $\mathrm{CO}_{2}$ decreased lightly rot incidence. Both cultivars showed good storability and might be stored for more than one month $m$ temperatures ranging from $-1,0^{\circ} \mathrm{C}$ to $+0,5^{\circ} \mathrm{C}$.

Key words: Vitis vinifera, post-harvest, cold storage.

\section{INTRODUÇÃO}

Nos últimos anos, tem-se expandido a área de cultivo de uvas de mesa no Brasil, e novas cultivares têm sido lançadas, buscando melhorar a qualidade dos frutos para consumo in natura. Cultivares tardias, como a Dona Zilá e a Tardia de Caxias, lançadas recentemente pelo CNPUVEMBRAPA, são alternativas para abastecimento do mercado após a comercialização das cultivares tradicionais do Rio Grande do Sul. No entanto, para se obter êxito na comercialização de uvas in natura, os frutos devem apresentar boa qualidade em nível

\footnotetext{
${ }^{1}$ Engenheiro Agrônomo, Professor do Departamento de Fototecnia, Universidade Federal de Santa Maria (UFSM), 97105-900, Santa

Maria-RS. Bolsista CNPq. E-mail: brackman@creta.ccr.ufsm.br. Autor para correspondência.

${ }^{2}$ Engenheiro Agrônomo, Aluno do Curso de Pós-Graduação em Agronomia, UFSM, Santa Maria - RS.

${ }^{3}$ Acadêmico do Curso de Agronomia, UFSM, Bolsista PIBIC/CNPq, Santa Maria - RS. 
de consumidor e as perdas pós-colheita devem ser baixas. No Brasil, essas perdas são elevadas, em tomo de 35\% (CENCI \& CHITARRA, 1994), e são caracterizadas pela perda de peso, escurecimento do ráquis, amolecimento das bagas, degrane e desenvolvimento de fungos causadores de podridões (NELSON, 1970; PERKINS-VEAZIE et al., 1992). Entretanto, alguns fatores, como a seleção de uvas, pré-resfriamento, tratamento com $\mathrm{SO}_{2}$, embalagem e condições de armazenamento podem contribuir na conservação da qualidade pós-colheita de uvas. (SAUNDERS \& TAKEDA, 1981; PERKINSVEAZIEtíaí,1992).

A temperatura de armazenamento varia conforme a cultivar, sendo que para uvas viníferas recomenda-se a temperatura de $-1,0$ a $-0,5^{\circ} \mathrm{C}$, e para uvas americanas de $-0,5$ a $0^{\circ} \mathrm{C}$ (HARDENBURG et al., 1986) e próximo a $-1,0^{\circ} \mathrm{C}$ para cultivares com alto teor de açúcares (FERRER \& TORRALLARDONA, 1970). Segundo CENCI (1994), as uvas desidratam pouco quando armazenadas a $-0,5^{\circ} \mathrm{C}$ e $95 \%$ de umidade relativa. A desidratação é o principal fator de degradação da qualidade de uva de mesa e se traduz em escurecimento do ráquis e degrane das bagas. Para diminuir as perdas por desidratação, a umidade relativa no armazenamento deve estar entre 90 e 95\% (SANTOS, 1993; BLEINROTH, 1984), sendo que umidade relativa próxima a $100 \%$ pode causar a ruptura da camada superficial de células das bagas, favorecendo o desenvolvimento de microorganismos (CHITARRA \& CHITARRA, 1990).

Para controle de microorganismos, vem sendo amplamente utilizado o dióxido de enxofre $\left(\mathrm{SO}_{2}\right)$, no entanto, fatores como a concentração do produto e o tempo de contato com os frutos são importantes para o controle de deteriorações (MITCHEL, 1990; SANTOS et al., 1996). O SO 2 , além de controlar fungos, influencia em fenómenos fisiológicos, diminuindo a atividade das oxidases, reduzindo o ritmo da respiração das uvas e diminuindo perdas de açúcares (GARCIA, 1967), além de diminuir o escurecimento do ráquis (NELSON, 1983; MANSOUR et al., 1984). Entretanto, o $\mathrm{SO}_{2}$ aplicado como fumigante em câmaras pode provocar uma ampla variação de respostas em função da cultivar, causando branqueamento de bagas e conferindo sabor estranho nas uvas (BALLINGER \& NESBITT, 1984). Devido ao resíduo de $\mathrm{SO}_{2}$ nas bagas e por ser nocivo à saúde humana, tratamentos alternativos têm sido avaliados na pesquisa internacional. A utilização de cloreto de cálcio $\left(\mathrm{CaCl}_{2} ;\right)$ em pré-colheita é uma dessas alternativas que pode manter a qualidade das uvas, prolongando o período pós-colheita (CENCI \&
CHITARRA, 1994; HEGAZI, 1997). A utilização de armazenamento em atmosfera controlada com altas concentrações de $\mathrm{CO}_{2}$ é outra prática em estudo em diversos países, visando a substituir a aplicação de $\mathrm{SO}_{2}$; nas câmaras frigoríficas no controle de podridões, devido aos seus efeitos no suprimento do crescimento de fungos como Penicillium expansum (BORTOLUZ et al., 1994).

O objetivo do presente trabalho foi avaliar o efeito de diferentes temperaturas, níveis de umidade relativa, aplicação de tratamentos póscolheita de $\mathrm{SO}_{2}, \mathrm{CO}_{2}$ e $\mathrm{CaCl}_{2}$ na manutenção da qualidade pós-colheita das uvas cvs. Dona Zilá e Tardia de Caxias.

\section{MATERIAL E MÉTODOS}

O experimento foi desenvolvido no Núcleo de Pesquisa em Pós-colheita (NPP) do Departamento de Fitotecnia da Universidade Federal de Santa Maria. As uvas foram colhidas em um parreiral comercial em início de produção, com idade de quatro anos, localizado no município de Farroupilha - RS e transportadas até o NPP, onde foi realizada uma seleção, descartando-se os cachos que apresentavam características de ataque de fungos ou lesões provocadas pelo transporte. Após a seleção, os cachos foram acondicionados em caixas de madeira de $10 \mathrm{~kg}$ e, logo após, armazenadas em minicâmaras experimentais, com volume de 232 litros.

O delineamento experimental utilizado foi o inteiramente casualizado, com três repetições e a unidade experimental composta por quatro cachos de uvas, com peso total de, aproximadamente, $\pm 1,4 \mathrm{~kg}$. Foram avaliados os tratamentos seguintes: [T1] $0,5^{\circ} \mathrm{C}$ com umidade relativa (UR) de $97 \%$; [T2] $-1,0^{\circ} \mathrm{C}$ com UR de $97 \%$; [T3] $-0,5^{\circ} \mathrm{C}$ com UR de 97\%; [T4] $-0,5^{\circ} \mathrm{C}$ com UR de $93 \%$; [T5] $-0,5^{\circ} \mathrm{C}$ com UR de $97 \%$ e $\mathrm{SO}_{2}$; [T6] $-0,5^{\circ} \mathrm{C}$ com UR de $97 \%$ e $20 \mathrm{kPa}$ de $\mathrm{CO}_{2}$ intermitente $(24 \mathrm{~h})$; [T7] $-0,5^{\circ} \mathrm{C}$ com UR de $97 \%$ e $5 \mathrm{kPa}$ de $\mathrm{CO}_{2}$ durante todo o período de armazenamento e; [T8] $-0,5^{\circ} \mathrm{C}$ com UR de $97 \%$ e $\mathrm{CaCl}_{2}$.

$\mathrm{O} \mathrm{SO}_{2}$ [T5] foi aplicado semanalmente na câmara, na dosagem de $4 \mathrm{~g}$ de metabissulfito de sódio $\left(\mathrm{Na}_{2} \mathrm{~S}_{2} \mathrm{O}_{5}\right)$, diluídos em $10 \mathrm{~m} \ell$ de água. $\mathrm{O}$ $\mathrm{Na}_{2} \mathrm{~S}_{2} \mathrm{O}_{5}$ em contato com a água volatiliza, liberando o $\mathrm{SO}_{2}$. O tratamento intermitente com $\mathrm{CO}_{2}$ [T6] consistiu da aplicação de $20 \mathrm{kPa}$ de $\mathrm{CO}_{2}$ por 24 horas semanalmente e, após o tratamento, a câmara foi arejada para a remoção do $\mathrm{CO}_{2} ; . \mathrm{O} \mathrm{CaCl}_{2}$; [T8] foi aplicado por imersão dos cachos numa solução de $0,5 \%$ de $\mathrm{CaCl}_{2}$ por dois minutos.

$\mathrm{O}$ monitoramento da temperatura foi realizado diariamente utilizando termómetros introduzidos na polpa de frutos. A umidade relativa foi aferida a cada três dias, com auxílio de um 
psicrômetro. Quando a umidade relativa estava $\pm 2 \%$ abaixo da desejada, nebulizava-se $50 \mathrm{~m} \ell$ de água no interior da câmara.

As condições de atmosfera controlada com alto $\mathrm{CO}_{2}$; [T6 e T7] foram estabelecidas com injeção desse gás nas câmaras, proveniente de cilindro de alta pressão. As concentrações de gases foram diariamente analisadas com um analisador eletrônico de $\mathrm{O}_{2}$ e $\mathrm{CO}_{2}$ de fluxo contínuo, marca Agridatalog. $\mathrm{O} \mathrm{O}_{2}$ consumido pela respiração dos frutos foi reposto através da injeção de ar nas câmaras e permaneceu sempre em concentrações maiores que $16 \%$. $\mathrm{O} \mathrm{CO}_{2}$; produzido foi absorvido com auxílio de uma solução de hidróxido de potássio (40\%), pela qual foi circulado o gás das câmaras.

As avaliações foram realizadas em dois momentos, na abertura das minicâmaras (35 dias de armazenamento) e após dois dias de exposição dos frutos à temperatura ambiente $\left( \pm 20^{\circ} \mathrm{C}\right)$, visando a simular o período de beneficiamento e comercialização. Avaliaram-se perda de peso, resistência da baga ao degrane, percentagem de degrane das bagas, escurecimento do ráquis, incidência de podridões, acidez titulável, sólidos solúveis totais (SST), além de uma análise sensorial.

Para determinação de perda de peso, as amostras foram pesadas no momento da instalação do experimento e na abertura das minicâmaras. Pela diferença de peso determinou-se a percentagem de perda de peso. A resistência da baga ao degrane foi determinada com um dinamômetro, através da aplicação de uma força sobre a baga até o seu desprendimento do ráquis. Os valores foram expressos em grama-força (gf). A percentagem de degrane foi calculada pelo número de bagas destacadas do ráquis sobre o número total de bagas do cacho. Para avaliação do escurecimento do ráquis foram estabelecidos três níveis: nível l, com até $10 \%$ do ráquis escurecido; nível 2 com 10 a $40 \%$ do ráquis escurecido e nível 3 , acima de $40 \%$ do ráquis escurecido. $\mathrm{O}$ escurecimento do ráquis foi expresso num índice, obtido pela média ponderada do nível de incidência de escurecimento do ráquis. $\mathrm{Na}$ avaliação de ocorrência de podridões, frutos com lesões características de ataque de patógeno foram considerados podres. A acidez titulável foi determinada através de titulação de $10 \mathrm{~m} \ell$ de suco, em $100 \mathrm{~m} \ell$ de água, com solução de $\mathrm{NaOH} 0,1 \mathrm{~N}$ até $\mathrm{pH} 8,1$. Os teores de SST foram determinados por refratometria. A avaliação sensorial foi realizada por um painel de cinco pessoas, avaliando a presença de sabores e/ou aromas estranhos nas uvas.

Os dados foram submetidos à análise de variância e as médias dos tratamentos comparadas estatisticamente pelo teste de Duncan em nível de
$5 \%$ de probabilidade de erro. Os dados expressos em percentagem foram transformados pela fórmula $\operatorname{arc.sen} . \sqrt{x / 100}$, antes da análise da variância.

\section{RESULTADOS E DISCUSSÕES}

Os resultados obtidos com as uvas cvs. Tardia de Caxias e Dona Zilá (Tabelas 1, 2, 3 e 4), demonstram que as duas cultivares apresentam boa capacidade de armazenamento. Após 35 dias de armazenamento refrigerado e dois dias em temperatura ambiente $\left( \pm 20^{\circ} \mathrm{C}\right)$, as uvas apresentavam-se em boas condições para o consumo. De modo geral, os SST mantiveram-se quase que inalterados durante o período de armazenamento, ocorrendo, no entanto, um decréscimo nos ácidos. Apesar da diminuição da acidez, a relação açúcar/acidez permaneceu satisfatória para o consumo de acordo com a avaliação sensorial. Esses resultados confirmam os de CAMARGO et $\boldsymbol{a l}$. (1996), que obtiveram boa qualidade de uvas 'Dona Zilá' após 30 dias de armazenamento.

A temperatura adequada de armazenamento das duas cultivares deve estar no intervalo de $-1,0^{\circ} \mathrm{C}$ a $0,5^{\circ} \mathrm{C}$, pois nas três temperaturas avaliadas, as uvas tiveram uma performance bastante semelhante e não sofreram danos fisiológicos, demonstrando que essas cultivares não são muito sensíveis a danos por baixa temperatura, como o escurecimento do ráquis e aumento da sensibilidade a podridões.

Durante o período de armazenamento, a perda de peso foi de 1 a $2 \%$ na condição com $\pm 97 \%$ de UR, porém com o decréscimo da UR para $\pm 93 \%$ houve uma maior perda de peso, além de um aumento considerável do degrane e do escurecimento do ráquis, quando as uvas foram expostas à temperatura ambiente. A maior perda de peso na UR de $93 \%$ deve-se à perda de água, o que, conseqüentemente, aumentou o escurecimento do ráquis e o degrane das bagas. Neste experimento, avaliou-se a baixa UR (93\%) com objetivo de propiciar um ambiente menos favorável ao desenvolvimento de patógenos, porém, essa redução na umidade não foi suficiente para reduzir a incidência de podridões nos frutos.

A utilização de $\mathrm{CaCl}_{2}$; em pós-colheita, ao contrário do que se esperava, aumentou a podridão dos frutos. Conforme CENCI \& CHITARRA (1994) e HEGAZI (1997), a utilização de $\mathrm{CaCl}_{2}$ em pré-colheita melhora a qualidade das uvas, no entanto, em pós-colheita, não foi observado benefícios neste experimento. Sugere-se que o $\mathrm{CaCl}_{2}$ pode ter removido a cerosidade natural das bagas ou causado danos às células da epiderme, predispondoas ao ataque de fungos. 
Tabela 1 - Perda de peso, resistência ao degrane, acidez titulável e teor de sólidos solúveis totais (SST) de uvas cv. Tardia de Caxias após 35 dias de armazenamento refrigerado, no momento da abertura das câmaras e mais dois dias de exposição à temperatura ambiente $\left( \pm 20^{\circ} \mathrm{C}\right)$. Santa Maria, 1998.

\begin{tabular}{|c|c|c|c|c|c|c|c|c|c|c|}
\hline \multicolumn{4}{|c|}{ TRATAMENTOS } & \multicolumn{4}{|c|}{ Abertura das câmaras } & \multicolumn{3}{|c|}{ Após dois dias } \\
\hline & $\begin{array}{l}\text { Temp. } \\
\left({ }^{\circ} \mathrm{C}\right)\end{array}$ & $\begin{array}{l}\text { UR } \\
(\%)\end{array}$ & & $\begin{array}{l}\text { Perda de } \\
\text { peso }(\%)\end{array}$ & $\begin{array}{c}\text { Resistência ao } \\
\text { degrane (gf) }\end{array}$ & $\begin{array}{l}\text { Acidez titulável } \\
\quad\left(\mathrm{cmol} . \mathrm{L}^{-1}\right)\end{array}$ & $\begin{array}{c}\text { SST } \\
\left({ }^{\circ} \mathrm{Brix}\right)\end{array}$ & $\begin{array}{c}\text { Resistência ao } \\
\text { degrane (gf) }\end{array}$ & $\begin{array}{l}\text { Acidez titulável } \\
\left(\mathrm{cmol} . \mathrm{L}^{-1}\right)\end{array}$ & $\begin{array}{c}\text { SST } \\
\text { ( }{ }^{\circ} \text { Brix) }\end{array}$ \\
\hline 1 & 0,5 & +97 & & - & $83,9 \mathrm{ab}$ & $6,52 \mathrm{bc}$ & $15,0 \mathrm{a}$ & 95,1 a & $6,35 \mathrm{~b}$ & $14,6 \mathrm{ab}$ \\
\hline 2 & $-1,0$ & +97 & & - & $88,1 \mathrm{ab}$ & $6,39 c$ & $15,3 \mathrm{a}$ & $70,3 \mathrm{bcd}$ & $6,59 a b$ & $15,0 \mathrm{ab}$ \\
\hline 3 & $-0,5$ & +97 & & $1,69 \mathrm{~b}^{3}$ & $117,4 \mathrm{a}$ & $6,57 \mathrm{bc}$ & $15,0 \mathrm{a}$ & $83,9 \mathrm{abc}$ & $6,11 b$ & $15,1 \mathrm{a}$ \\
\hline 4 & $-0,5$ & +93 & & $3,06 \mathrm{a}$ & $62,2 \mathrm{~b}$ & $6,74 a b c$ & $15,2 \mathrm{a}$ & $88,0 \mathrm{ab}$ & $6,78 \mathrm{ab}$ & $15,1 \mathrm{a}$ \\
\hline 5 & $-0,5$ & +97 & $+\mathrm{SO}_{2}$ & - & $103,1 \mathrm{ab}$ & $7,20 \mathrm{ab}$ & $14,3 \mathrm{a}$ & $66,0 \mathrm{bcd}$ & $6,35 b$ & $14,3 \mathrm{~b}$ \\
\hline 6 & $-0,5$ & +97 & $+20 \mathrm{kPa} \mathrm{CO} / 24 \mathrm{~h}^{1}$ & - & $96,3 \mathrm{ab}$ & $7,26 \mathrm{a}$ & $15,1 \mathrm{a}$ & $56,5 \mathrm{~d}$ & $7,24 \mathrm{a}$ & $14,6 \mathrm{ab}$ \\
\hline 7 & $-0,5$ & +97 & $+5 \mathrm{kPa} \mathrm{CO}_{2}^{2}$ & - & $86,8 \mathrm{ab}$ & $6,51 b c$ & $14,7 \mathrm{a}$ & $48,4 d$ & $6,49 b$ & $14,5 \mathrm{ab}$ \\
\hline 8 & $-0,5$ & +97 & $+\mathrm{CaCl}_{2}$ & - & $116,8 \mathrm{a}$ & $7,32 \mathrm{a}$ & $14,7 \mathrm{a}$ & $61,2 \mathrm{~cd}$ & $6,37 b$ & $14,6 a b$ \\
\hline \multicolumn{4}{|c|}{$\mathrm{CV}(\%)$} & 9,5 & 23,57 & 5,39 & 19,49 & 17,34 & 5,95 & 2,75 \\
\hline
\end{tabular}

$20 \mathrm{kPa}$ de $\mathrm{CO}_{2}$ aplicados durante $24 \mathrm{~h}$, semanalmente;

${ }^{2} 5 \mathrm{kPa}$ de $\mathrm{CO}_{2}$ durante todo o período de armazenamento.

${ }^{3}$ Médias seguidas pela mesma letra na vertical não diferem pelo teste de Duncan em nível de $5 \%$ de probabilidade de erro.

$\mathrm{O}$ tratamento com $\mathrm{SO}_{2}$, de modo geral, não apresentou eficiência no controle de podridões, como citam alguns autores (KLAYTON, 1982; TAYLOR \& CHAMBERS, 1990). Porém, deve-se levar em consideração que a incidência de podridões foi baixa em todos os tratamentos. Isso deve-se, possivelmente, à eliminação de bagas danificadas no momento do armazenamento, além do curto período de conservação, associado a condições pouco favoráveis para o desenvolvimento de fungos do género Botrytís, principal agente causal observado no experimento.
$\mathrm{Na}$ condição de $5 \mathrm{kPa}$ de $\mathrm{CO}_{2}$, durante todo o período de armazenamento, ocorreu uma redução nas podridões nas duas cultivares avaliadas, sendo que com a aplicação semanal de $20 \mathrm{kPa}$ de $\mathrm{CO}_{2}$ por $24 \mathrm{~h}$, a redução da podridão ocorreu somente na cultivar Dona Zilá. Esses efeitos positivos da alta concentração de $\mathrm{CO}_{2}$ são devidos ao estabelecimento de um ambiente desfavorável para o desenvolvimento dos fungos $\mathrm{e}$, provavelmente, a uma redução do metabolismo dos frutos. Os fungos, em geral, são sensíveis às altas concentrações de $\mathrm{CO}_{2}$; (TABAK \& COOKE, 1968).

Tabela 2 - Percentagem de degrane, índice de escurecimento do ráquis e incidência de podridões em uvas cv. Tardia de Caxias após 35 dias de armazenamento refrigerado, no momento da abertura das câmaras e mais dois dias de exposição à temperatura ambiente $\left( \pm 20^{\circ} \mathrm{C}\right)$. Santa Maria, 1998 .

\begin{tabular}{|c|c|c|c|c|c|c|c|c|c|}
\hline \multicolumn{4}{|c|}{ TRATAMENTOS } & \multicolumn{3}{|c|}{ Abertura das câmaras } & \multicolumn{3}{|c|}{ Após dois dias } \\
\hline & Temp. $\left({ }^{\circ} \mathrm{C}\right)$ & UR $(\%)$ & & $\begin{array}{c}\text { Degrane } \\
(\%)\end{array}$ & $\begin{array}{l}\text { Escurecimento } \\
\text { do ráquis } \\
(1-3)\end{array}$ & $\begin{array}{l}\text { Podridāo } \\
\text { (\%) }\end{array}$ & $\begin{array}{c}\text { Degrane } \\
(\%)\end{array}$ & $\begin{array}{l}\text { Escurecimento } \\
\text { do ráquis } \\
(1-3)\end{array}$ & $\begin{array}{c}\text { Podridão } \\
(\%)\end{array}$ \\
\hline $\begin{array}{l}1 \\
2 \\
3 \\
4 \\
5 \\
6 \\
7 \\
7 \\
8\end{array}$ & $\begin{array}{r}0,5 \\
-1,0 \\
-0,5 \\
-0,5 \\
-0,5 \\
-0,5 \\
-0,5 \\
-0,5\end{array}$ & $\begin{array}{l}+97 \\
+97 \\
+97 \\
+93 \\
+97 \\
+97 \\
+97 \\
+97\end{array}$ & $\begin{array}{l}+\mathrm{SO}_{2} \\
+20 \mathrm{kPa} \mathrm{CO}_{2} / 24 \mathrm{~h}^{1} \\
+5 \mathrm{kPa} \mathrm{CO}_{2}^{2} \\
+\mathrm{CaCl}_{2}\end{array}$ & $\begin{array}{l}3,53 \mathrm{a}^{3} \\
1,89 \mathrm{a} \\
4,39 \mathrm{a} \\
4,45 \mathrm{a} \\
2,28 \mathrm{a} \\
2,58 \mathrm{a} \\
2,03 \mathrm{a} \\
4,91 \mathrm{a}\end{array}$ & $\begin{array}{l}1,83 \mathrm{~b} \\
2,17 \mathrm{~b} \\
1,92 \mathrm{~b} \\
2,83 \mathrm{a} \\
1,92 \mathrm{~b} \\
2,08 \mathrm{~b} \\
1,25 \mathrm{c} \\
2,08 \mathrm{~b}\end{array}$ & $\begin{array}{l}1,90 \mathrm{bc} \\
1,15 \mathrm{c} \\
3,07 \mathrm{ab} \\
3,13 \mathrm{ab} \\
1,69 \mathrm{bc} \\
1,96 \mathrm{bc} \\
0,00 \mathrm{~d} \\
3,98 \mathrm{a}\end{array}$ & $\begin{array}{l}5,5 \mathrm{ab} \\
4,3 \mathrm{ab} \\
2,5 \mathrm{ab} \\
6,2 \mathrm{a} \\
4,5 \mathrm{ab} \\
1,9 \mathrm{~b} \\
5,0 \mathrm{ab} \\
6,1 \mathrm{a}\end{array}$ & $\begin{array}{l}1,17 \mathrm{~cd} \\
1,42 \mathrm{ab} \\
0,83 \mathrm{e} \\
1,50 \mathrm{a} \\
1,08 \mathrm{~d} \\
1,25 \mathrm{bcd} \\
1,17 \mathrm{~cd} \\
1,33 \mathrm{abc}\end{array}$ & $\begin{array}{l}1,5 \mathrm{c} \\
0,9 \mathrm{~cd} \\
1,4 \mathrm{~cd} \\
0,6 \mathrm{~cd} \\
4,1 \mathrm{~b} \\
1,1 \mathrm{~cd} \\
0,6 \mathrm{~d} \\
8,8 \mathrm{a}\end{array}$ \\
\hline \multicolumn{4}{|c|}{$\mathrm{CV}(\%)$} & 18,70 & 10,15 & 17,19 & 21,86 & 10,25 & 12,09 \\
\hline
\end{tabular}

$20 \mathrm{kPa}$ de $\mathrm{CO}_{2}$ aplicados durante $24 \mathrm{~h}$, semanalmente;

${ }^{2} 5 \mathrm{kPa}$ de $\mathrm{CO}_{2}$ durante todo o período de armazenamento.

${ }^{3}$ Médias seguidas pela mesma letra na vertical não diferem pelo teste de Duncan em nível de $5 \%$ de probabilidade de erro. 
Tabela 3 - Perda de peso, resistência ao degrane, acidez titulável e teor de sólidos solúveis totais (SST) de uvas cv. Dona Zilá após 35 dias de armazenamento refrigerado, no momento da abertura das câmaras e mais dois dias de exposição à temperatura ambiente ( $\pm 20^{\circ} \mathrm{C}$ ). Santa Maria, 1998.

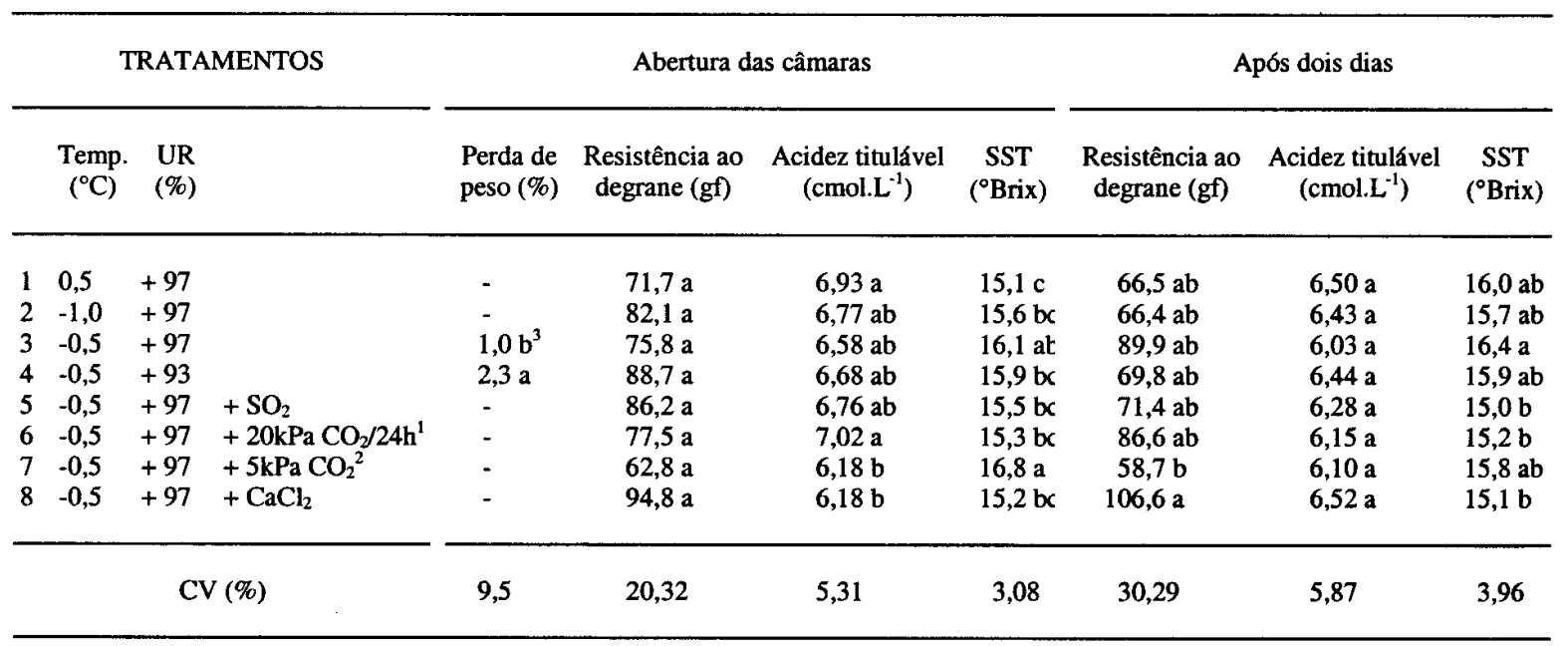

${ }^{1} 20 \mathrm{kPa}$ de $\mathrm{CO}_{2}$ aplicados durante $24 \mathrm{~h}$, semanalmente;

${ }^{2} 5 \mathrm{kPa}$ de $\mathrm{CO}_{2}$ durante todo o período de armazenamento.

${ }^{3}$ Médias seguidas pela mesma letra na vertical não diferem pelo teste de Duncan em nível de $5 \%$ de probabilidade de erro.

Segundo AGAR et al. (1990), 25 a 30\% de $\mathrm{CO}_{2}$ inibem totalmente o crescimento de Botrytís na temperatura de $1^{\circ} \mathrm{C}$. Também deve ser considerado que o aumento na concentração de $\mathrm{CC}>2$ inibe podridões devido ao efeito do $\mathrm{CO}_{2}$ sobre o retardamento do processo de amadurecimento e senescência, o qual torna os frutos mais sensíveis ao ataque de patógenos (BRACKMANN et al., 1996). Porém, na avaliação da viabilidade do uso da atmosfera controlada para uvas deve-se levar em consideração o custo da instalação da mesma e o curto período de armazenamento. $\mathrm{Na}$ avaliação sensorial, não foram constatados sabores e/ou aromas estranhos nos frutos em função dos tratamentos.

\section{CONCLUSÕES}

As cultivares de uvas de mesa 'Tardia de Caxias' e 'Dona Zilá' possuem boa capacidade de

Tabela 4 - Percentagem de degrane, índice de escurecimento do ráquis e incidência de podridões em uvas cv. Dona Zilá após 35 dias de armazenamento refrigerado, no momento da abertura das câmaras e mais dois dias de exposição à temperatura ambiente $\left( \pm 20^{\circ} \mathrm{C}\right)$. Santa Maria, 1998.

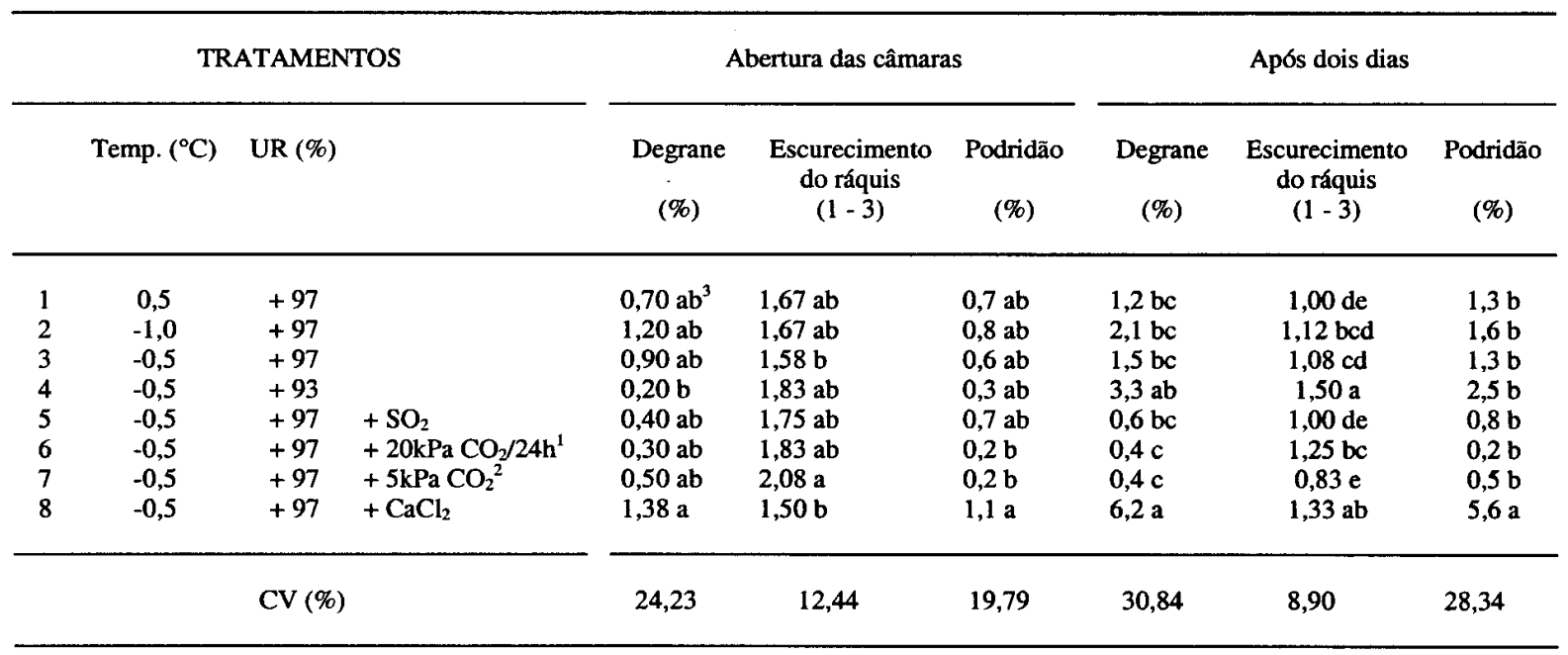

\footnotetext{
' $20 \mathrm{kPa}$ de $\mathrm{CO}_{2}$ aplicados durante $24 \mathrm{~h}$, semanalmente;

${ }^{2} 5 \mathrm{kPa}$ de $\mathrm{CO}_{2}$ durante todo o período de armazenamento.

${ }^{3}$ Médias seguidas pela mesma letra na vertical não diferem pelo teste de Duncan em nível de $5 \%$ de probabilidade de erro.
} 
armazenamento, podendo ser mantidas sob refrigeração, na temperatura de $-1,0^{\circ} \mathrm{C}$ a $+0,5^{\circ} \mathrm{C}$, por um período de 30 dias. A alta umidade relativa (97\%) diminui a perda de peso, o escurecimento do ráquis e o degrane das bagas. A aplicação póscolheita de $\mathrm{CO}_{2}$; reduz a incidência de podridões nas uvas. Por outro lado, o SÓ; não apresenta efeito na supressão ao desenvolvimento de fungos. A aplicação pós-colheita de $\mathrm{CaCl}_{2}$, não é recomendada para uvas.

\section{AGRADECIMENTOS}

Os autores agradecem à FAPERGS, ao PIBIC/CNPq e às Prefeituras Municipais da Serra Gaúcha pelo financiamento do projeto.

\section{REFERÊNCIAS BIBLIOGRÁFICAS}

AGAR, T., GARCIA, J. M., WIEDTKE, V., et al. Effect of high $\mathrm{CO}_{2}$ and low $\mathrm{O}$; concentrations on the growth of Botrytis cinerea at different temperatures. Gartenbauwiissenshaf, Sttutgart, v.55, n.5, p.219-222,1990.

BALLINGER, W.E., NESBITT, W.B. Quality of Euvitis hibrid Bunch. grapes after low temperature storage with sulfur dioxide. HortiScience, Alexandria, v.109, n.6, p.831-834, 1984.

BLEINROTH. E.W. Curso de frigoconservação de frutas Campinas : ITAL, 1984. 138p.

BORTOLUZ, L., BREDEMEIR, F. D., MAZARO, S. M. et a Efeito do $\mathrm{C} 02$ e 02 na supressão do crescimento de Peniciüium expansum (Link). In: JORNADA INTEGRADA DE PESQUISA EXTENSÃO E ENSINO, 1, 1994, Santa Maria, RS. Anais... Santa Maria : Pró-reitoria de Pósgraduação e Pesquisa, 1994. 775p. p.309.

BRACKMANN, A., SAQUET, A.A., VEIGA, V.V., et al. Efeito das concentrações de $\mathrm{CO}_{2}$ e $\mathrm{O}_{2}$ no crescimento e esporulação de Penicillium expansun (Link) Thom, in vitro. Revista Brasileira de Agrociência, Pelotas, v.2, n.3, p.147-150,1996.

CAMARGO, V.A., CZERMADMSKI, A.B.C., SÔNEGO, O.R et $\boldsymbol{a}$. Influência da embalagem e dióxido de enxofre na frigoconservação de uva Dona Zilá. to: CONGRESSO BRASILEIRO DE VITICULTURA E ENOLOGIA, 8, 1996 , Bento Gonçalves, RS. Resumos... Bento Gonçalves : EMBRAPA-CNPUV, 1996. p.69.

CENCI, S.A. Ácido naftalenoacético (ANA) e cloreto de cálcio na pré-colheita de uva niágara rosada (Vitis labrusca $\mathrm{L}$. $\mathrm{X}$ Vitis vinifera L.): Avaliação do potencial de conservação no armazenamento. Lavras, MG, 1994. 129p. Tese (Doutorado em Agronomia) - Curso de Pós-graduação em Ciência dos Alimentos, Escola Superior de Agricultura de Lavras, 1994.

CENCI, S.A., CHITARRA, M.I.F. Controle da abscisão póscolheita de uva 'Niágara Rosada' Vitis (labrusca L. $x$ vinifera L.): mecanismos decorrentes da aplicação de ANA e cálcio no campo. Revista Brasileira de Fruticultura, Cruz das Almas, v. 16, n.l,p.146-155,1994.

CHITARRA, M.I.F., CHIT ARRA, A.B. Pós-colheita de frutos e hortaliças: Fisiologia e manuseio. Lavras : ESAL-FAEPE, 1990. 320p.
FERRER, M.M., TORRALLARDONA, S.D. Frigoconservacion y manejo, frutos, flores y hortaliças. Madrid:Aedos, 1970 p. $13-160$

GARCIA, A.D. El empleo dei anidrido sulfuroso em Ia comercializacion de Ia uva de mesa. Madrid : Ministério da Agricultura, 1967. 129p. (Monografia, 17).

HARDENBURG, R.E., WATADA, A.E., WANG, C.Y. The commercial storage of iruits, vegetables and florist and nursery stocks. Washington : USDA, 1986. 130p. (Agriculture Handbook, 66).

HEGAZI, A. Effect of spraying potassium and calcium on the shelf-life of flame seedless grapes. ta: BNTERNATIONAL SYMPOSIUM ON EFFECT OF PREHARVEST AND POSTHARVEST FACTORS ON STORAGE OF FRUIT, 1997, Warsaw, Poland. Abstracts... Warsaw, Poland : Research Institui of Pomology and Horiculture, 1997. 81p. p.61.

KLAYTON, E.N. Retarding Deterioration of table grapes with inpackage surfur dioxide generators with and without refrigeration. ta: INTERNATIONAL HORTICULTURAE CONGRESS, 21, 1982, Hamburg. Abstract... Hamburg : Intemational Society for Horticultural Science, 1982. v. 1. p.1176.

MANSOUR, K.M., ZEINAB, M., TOBSHY, E.L, et al. Effect of in - package $\mathrm{SO}_{2}$ - gerador on postharvest decay and quality of banati grapes. Egiptian Journal of Horticulturae, Cairo, v.11,n.11,p.12-18,1984.

MITCHEL, F.G. Postharvest handüng of table grapes. Stratcgies of fruits handling and storage. Davis : USDA. 1990.3p.

NELSON, K.E. Packging and handüng trials on export of grapes. BlueAnchor, Sacramento, v.47, p.9-13,1970.

PERKINS-VEAZIE, P.M., COLLINS, J.K., LLOYD, J., et al Influence of package on post-harvest quality of Oklalioma and Arkansas table grapes. American Journal Enology Vitículture, Davis, v.43, n. 1, p.79-82,1992.

SANTOS, E.J.R. Frigoconservação da uva cv. Itália (Vitis vinifera L.) com diferentes embalagens e uso de SO $_{2}$;.Pelotas, RS, 1994. 43p. Dissertação (Mestrado em Agronomia) - Curso de Pós-graduação em Agronomia, Universidade Federal de Pelotas, 1994.

SANTOS, E.J.R., FERREIRA, A.A., BILHALVA, A.B. Controle de podridões em uvas 'Itália' (Vitis vinifera L.) em armazenamento refrigerado. Revista Brasileira de Fruticultura, Cruz das Almas, v.18, n.1, p.147-149,1996.

SAUNDERS, M.S., TAKEDA, F. Postharvest physiology and senescence in Muscadines. Proceedmgs of the Florida of State Horticultural Society, Tallahasse, v.94, p.340-343, 1981.

TABAK, H.H., COOKE, W.M.B. The effects of gaseous environments on the growth and metabolism of fung. Botanik Review, v.34, p. 126-252,1968.

TAYLOR, M.A., CHAMBERS, K.R. Effect of temperature, $\mathrm{SO}_{2}$ concentation and exposure time on storage quality of Waltham Cross and Barlink grapes. Deciduous fruit Grower. Sagtevrugteboer, v.40, n.9, p.327-331,1990. 